\title{
THE $f$-DECOMPOSITION OF ARTINIAN MODULES OVER HYPERFINITE GROUPS
}

\author{
by Z. Y. DUAN
}

(Received 29th May 1993)

\begin{abstract}
A $\mathbb{Z} G$-module $A$ is said to have an $f$-decomposition if $A=A^{f} \oplus A^{\jmath}$ in which $A^{f}$ is a $\mathbb{Z} G$-submodule of $A$ such that each irreducible $\mathbb{Z} G$-factor of $A^{\mathcal{S}}$ as an abelian group is finite and the $\mathbb{Z} G$-submodule $A^{\mathcal{J}}$ has no finite irreducible $\mathbb{Z} G$-factors. In this paper, we prove that: if $G$ is a hyperfinite group then any artinian $\mathbb{Z} G$-module $A$ has an $f$-decomposition, which gives a positive answer to the question raised by D.I. Zaitzev in 1986.
\end{abstract}

1991 Mathematics subject classification: 20F19, $20 \mathrm{C07}$

If $G$ is a locally soluble hyperfinite group, then it was known that any minimal (or artinian), maximal (or noetherian), or minimax $\mathbb{Z} G$-module $A$ has an $f$-decomposition, that is, $A=A^{\mathcal{S}} \oplus A^{\bar{f}}$ in which $A^{\mathcal{S}}$ is a $\mathbb{Z} G$-submodule of $A$ such that each irreducible $\mathbb{Z} G$-factor of $A^{f}$ as an abelian group is finite and the $\mathbb{Z} G$-submodule $A^{\bar{f}}$ of $A$ has no finite irreducible $\mathbb{Z} G$-factors (see [1, 2, and 3]). In the above results, is the locally soluble condition necessary? This question was asked by Zaitzev in 1986 [3]; we give a positive answer for the artinian case. That is, we prove the following

Theorem. If $G$ is a hyperfinite group, then any artinian $\mathbb{Z} G$-module $A$ has an $f$-decomposition.

In order to prove the theorem, we need some lemmas.

Lemma 1. Let $G$ be a group, $x$ an element of $G, A$ a $\mathbb{Z} G$-module with $p A=0$ for some prime $p$, and $B$ a nonzero subset of $A$. If also $x$ is of order $p$, then $B(x-1) \neq B$.

Proof. Suppose $B(x-1)=B$ then for $0 \neq a \in B$ we have

$$
\begin{aligned}
a & =a_{1}(x-1)=a_{2}(x-1)^{2}=\cdots=a_{m}(x-1)^{p} \\
& =a_{m}\left[\sum_{i=0}^{p}\left(\begin{array}{c}
p \\
i
\end{array}\right)(-1)^{i} x^{p-i}\right]=a_{m}\left(x^{p}-1\right)=0,
\end{aligned}
$$

a contradiction.

The following lemma, though easy, is the key to our removal of the solubility 
hypothesis. We emphasise that $S$ is assumed only to be a normal subset; that is, $S^{g}=S$ for all $g \in G$.

Lemma 2. Let $G$ be a group, $S$ a normal subset of $G$ and $A$ a $\mathbb{Z} G$-module. Then $C_{A}(S)$ and $[A, S]=\sum_{x \in S} A(x-1)$ are $\mathbb{Z} G$-submodules of $A$.

The proofs of Lemma 2 and the well-known Lemma 3 are left to the reader.

Lemma 3. Let $G$ be a group, $x$ an element of $G$, and $H$ a subgroup of $G$ contained in the centralizer $C_{G}(x)$ of $x$ in $G$. If $A$ is a $\mathbb{Z} G$-module, then for any $\mathbb{Z} H$-submodule $B$ of $A$ we have $B(x-1)$ and $C_{B}(x)$ are $\mathbb{Z} H$-submodules of $A$ and $B / C_{B}(x) \cong{ }_{2 H} B(x-1)$.

Lemma 4. Let $G$ be a hyperfinite group and $A$ an artinian $\mathbb{Z} G$-module such that $p A=0$ for some prime $p$. If the irreducible $\mathbb{Z} G$-factors of $A$ as abelian groups are all finite, then $A$ is finite.

Proof. Suppose $A$ is infinite, then by the artinian condition we may assume each proper $\mathbb{Z} G$-submodule of $A$ is finite. We may also assume that $G$ acts faithfully on $A$. If $G$ were finite then $A$ would be artinian as a $\mathbb{Z}$-module and so would be finite, therefore we may assume that $G$ is infinite.

Let $B$ be a proper $\mathbb{Z} G$-submodule of $A$ and let $H=C_{G}(B)$, then $H$ is a normal subgroup of finite index in $G$. So $H$ contains a nontrivial finite subgroup $K$ which is normal in $G$ (by $G$ being hyperfinite). Let $q$ be a prime factor of the order of $K$ and let $S=\{x \in K ; x$ is of order $q\}$. For $G_{1}=C_{G}(S)$, since $G_{1} \geqq C_{G}(K)$ and $G / C_{G}(K)$ is finite so $G_{1}$ is a subgroup of finite index in $G$. Thus $A$ is an infinite artinian $\mathbb{Z} G_{1}$-module. Therefore $A$ contains a least $\mathbb{Z} G_{1}$-submodule $A_{1}$ such that $A_{1}$ is infinite, $B<A_{1}$, and each proper $\mathbb{Z} G_{1}$-submodule of $A_{1}$ as an abelian group is finite. Since $C_{A}(S)$ is a $\mathbb{Z} G$-submodule of $A$ (Lemma 2) and $B \leqq C_{A}(S)<A$ so $C_{A}(S)$ as an abelian group is finite. Thus $C_{A_{1}}(S)=$ $A_{1} \cap C_{A}(S)$ is a proper $\mathbb{Z} G_{1}$-submodule of $A_{1}$ and $B \leqq C_{A_{1}}(S)$. Hence there exists $x_{0} \in S$ such that $B \leqq C_{A_{1}}\left(x_{0}\right)<A_{1}$. Let $G_{2}=C_{G}\left(x_{0}\right)$, then $G_{2} \geqq G_{1}$ and so $G_{2}$ is of finite index in $G$. Clearly there exists a least $\mathbb{Z} G_{2}$-submodule $A_{2}$ of $A$ such that $A_{2}$ is an infinite artinian $\mathbb{Z} G_{2}$-module, each proper $\mathbb{Z} G_{2}$-submodule of $A_{2}$ as an abelian group is finite, and $A_{1}$ is contained in $A_{2}$. For $x_{0} \in G_{2}$, if $\left|x_{0}\right|=q \neq p$ then, by Fitting's lemma, $A_{2}=\left[A_{2},<x_{0}>\right]+C_{A_{2}}\left(<x_{0}>\right)$. Since $B \leqq C_{A_{1}}\left(x_{0}\right)<A_{1}$ so $B \leqq C_{A_{2}}\left(<x_{0}>\right)<A_{2}$ and then $\left[A_{2},<x_{0}>\right]$ and $C_{A_{2}}\left(<x_{0}>\right)$ are nonzero proper $\mathbb{Z} G_{2}$-submodules of $A_{2}$. Thus $\left[A_{2},\left\langle x_{0}\right\rangle\right)$ are finite and so $A_{2}$ is finite, a contradiction. Therefore $\left|x_{0}\right|=q=p$. Since $B \leqq C_{A_{1}}\left(x_{0}\right)<A_{1}$ and $A_{2}\left(x_{0}-1\right)<A_{2}$ (Lemma 1), we have $B \leqq C_{A_{2}}\left(x_{0}\right)<A_{2}$ and so both $C_{A_{2}}\left(x_{0}\right)$ and $A_{2} / C_{A_{2}}\left(x_{0}\right) \cong{ }_{2 G_{2}}\left(A_{2}\left(x_{0}-1\right)\right)$ are finite. Hence $A_{2}$ is finite, a contradiction again. Thus the result holds.

Lemma 5. If $G$ is a hyperfinite group and $A$ an artinian $\mathbb{Z} G$-module all of whose irreducible $\mathbb{Z} G$-factors are finite, then $A$ as an abelian group is Cernikov and $G / C_{G}(A)$ is finite. 
Proof. This is similar to the proof of Theorem 3 in [2].

Proof of Theorem. Suppose $A$ does not have an $f$-decomposition, then by the artinian condition we may assume that each proper $\mathbb{Z} G$-submodule of $A$ has one. Since $G$ is locally finite and $A$ is artinian, $A$ is periodic and further $A$ is a $p$-group for some prime $p$. Let $M$ be the sum of all proper $\mathbb{Z} G$-submodules of $A$; then $M$ has an $f$-decomposition and so $M \neq A$. Thus $M$ is the unique maximal submodule of $A$. We may assume that $G$ acts faithfully on $A$ and consider the irreducible $\mathbb{Z} G$-image $A / M$. (1) If $A / M$ is finite then we may assume $M=M^{f}$ by investigating $A / M^{f}$; similarly, (2) if $A / M$ is infinite we assume $M=M^{f}$. Also, it is clear that $G$ is infinite.

Case 1. $A / M$ is finite and $M=M^{\overline{\mathcal{S}}}$.

In this case, let $H=C_{G}(A / M)$, then $G / H$ is finite and so $H$ contains a nontrivial finite subgroup $K$ which is normal in $G$. Let $q$ be a prime factor of the order of $K$ for some prime $q$ and let $S=\{x \in K ; x$ is of order $q\}$. For $G_{1}=C_{G}(S)$, we have $G_{1} \geqq C_{G}(K)$ and so $G_{1}$ is of finite index in $G$. Therefore $A$ is an artinian $\mathbb{Z} G_{1}$-module and then $A$ contains a least $\mathbb{Z} G_{1}$-submodule $A_{1}$ such that $A_{1}$ is not contained in $M$. Then $A_{1} / A_{1} \cap$ $M)\left(\cong_{Z G_{1}}\left(A_{1}+M\right) / M\right)$ is a finite irreducible $\mathbb{Z} G_{1}$-module. For $x \in S$, since $S \leqq K \leqq H=$ $C_{G}(A / M)$ we have $A_{1} / C_{A_{1}}(x)\left(\cong \mathbb{Z G}_{1} A_{1}(x-1) \leqq M\right)$ has no nonzero finite $\mathbb{Z} G_{1}$-factors and then $C_{A_{1}}(x)$ is not contained in $M$. Thus $C_{A_{1}}(x)=A_{1}$ for any $x \in S$ and then $C_{A_{1}}(S)=A_{1}$, which shows that $A_{1} \leqq C_{A}(S)$. So the $\mathbb{Z} G$-submodule $C_{A}(S)$ of $A$ is not contained in $M$ and then $C_{A}(S)=A$, which is contrary to $G$ being faithful on $A$. Case 1 is proved.

Case 2. $A / M$ is infinite and $M=M^{f}$.

In this case, $M$ as an abelian group is Črnikov and $G / C_{G}(M)$ is finite, by Lemma 5. Consider $A$ as a $\mathbb{Z} G_{1}$-module, where $G_{1}=C_{G}(M)$. Then $A$ is artinian and so contains a least $\mathbb{Z} G_{1}$-submodule $A_{1}$ which is not contained in $M$. As $G_{1}$ contains a nontrivial finite subgroup $F$ which is normal in $G$ so we must have $A_{1} \neq C_{A_{1}}(F)$, since otherwise $A_{1} \leqq C_{A}(F)$ would imply that $C_{A}(F)=A$, contrary to the faithfulness of $G$. Since $C_{A_{1}}(F) \geqq M_{1}\left(=A_{1} \cap M\right)$ and $A_{1} / M_{1}\left(\cong Z_{G 1}\left(A_{1}+M\right) / M\right)$ is irreducible, $C_{A_{1}}(F)=M_{1}$. Let $G_{2}=C_{G_{1}}(F)$, then $G_{1} / G_{2}$ is finite and then $A_{1}$ is an artinian $\mathbb{Z} G_{2}$-module. Let $A_{2}$ be a least $\mathbb{Z} G_{2}$-submodule of $A_{1}$ not contained in $M$. By $C_{A_{1}}(F)=M_{1}$ we have $C_{A_{2}}(F)=$ $M_{2}\left(=A_{2} \cap C_{A_{1}}(F)=A_{2} \cap M_{1}=A_{2} \cap A_{1} \cap M=A_{2} \cap M\right)$, it implies that there exists $x_{0} \in F$ such that $A_{2} \neq C_{A_{2}}\left(x_{0}\right)\left(\geqq M_{2}\right)$. Therefore $A_{2}\left(x_{0}-1\right)\left(\cong_{\mathrm{ZG}_{2}} A_{2} / C_{A_{2}}\left(x_{0}\right)\right)$ is an infinite irreducible $\mathbb{Z} G_{2}$-submodule of $A_{2}$ and then of $A$. Thus $A$ contains an infinite irreducible $\mathbb{Z} G$-submodule generated by $A_{2}\left(x_{0}-1\right)$ and then $A$ has an $f$-decomposition, a contradiction. Case 2 is proved.

By the above proof, the theorem is true.

Acknowledgement. I would like to give my thanks to Dr M. J. Tomkinson and, for financial support, to the Chinese State Education Committee and the Science Committee of Chongqing. 


\section{REFERENCES}

1. Z. Y. Duan, Noetherian modules over hyperfinite groups, (Ph.D thesis, University of Glasgow, 1991).

2. Z. Y. DUAN and M. J. Tomkinson, The decomposition of minmax modules over hyperfinite groups, Arch. Math. 61 (1993), 340-343.

3. D. I. ZaITZEv, Splitting of extensions of abelian groups, Akad. Nauk Ukrain. SSR, Inst. Mat. Kiev (1986), 22-31.

Department of Mathematics

SOUTHWEST Teachers University

Chongeing, 630715

P.R. CHINA 